\title{
A WEB-BASED 3D SIMULATION PLATFORM AIMED AT POLICY MAKERS FOR ESTIMATING THE EFFECTS OF URBAN HEAT ISLANDS
}

\author{
Gabriel WURZER ${ }^{1}$, Ulrich PONT ${ }^{2 *}$, Wolfgang E. LORENZ ${ }^{1}$, Sigrun SWOBODA ${ }^{1}$, Ardeshir MAHDAVI ${ }^{2}$
}

\section{Abstract}

The prediction of microclimate effects within an urban context through simulations has previously been done through elaborate software packages that offer accurate results but require extensive domain knowledge and time for the preparation of a model. In day-to-day policymaking, however, these efforts may turn out to be a major limiting factor: Policymakers and city administrators need a quick tool that can predict the effects of urban interventions in a variety of possible scenarios rather than a full-fledged simulation study for a limited set of specific interventions. In our work, we have been seeking to address this mismatch in expectations by using the best of both worlds: We have approximated results from a full-fledged simulation that uses a Cellular Automaton running within a $3 D$ city visualization. Policymakers can edit the urban context by changing cell types (placement of greenery, etc.), thus leading to scenarios that can be compared to the status quo. As a matter of fact, policymakers can use these comparisons to argue for measures that mitigate the effects of heat islands, without requiring extensive domain knowledge or the effort to prepare a simulation model.

\section{INTRODUCTION}

Climate change affects urban areas to a greater extent than the countryside: the average differences in temperature are already in a range of 3 degrees during the day and more than 10 degrees in the night during the summer and winter (U.S. Envirnment Protection Agency 2019); however, such effects are highly location-dependent ("urban heat islands" ("UHI"), "street canyon effect"). Predictions of microclimate effects within the urban context through simulations have previously been done through elaborate software packages such as ENVI-met (Bruse 1999, Bruse and Fleer 1998) (see "Background" section), which offer accurate results but require extensive domain knowledge and time for the preparation of the model. Ironically, policymakers and city administrators might have neither, which

\section{Address}

1 Dept. of Digital Architecture and Planning, TU Wien, Karlsplatz 13, 1040 Vienna, Austria

1 Dept. of Building Physics and Building Ecology, TU Wien, Karlsplatz 13, 1040 Vienna, Austria

* Corresponding author: ulrich.pont@tuwien.ac.at

\section{Key words}

- Pixel/Voxel based approach,

- Summer overheating,

- Mitigation,

- Urban greenery,

- Decision support. 
- A 2D Cellular Automaton that runs within that visualization and approximates a precomputed urban climate simulation with three cell types (sealed, greenery, water) and their rules for updating (see "Cellular Automaton" section).

- These rules for updating need to be calibrated in such a way that the Cellular Automaton produces values close to what a full simulation would produce, albeit at minimal computational expense. The "Approximating Simulated Data - Vienna Case Study" section provides an example of such a calibration step.

In the final step, the user (i.e., the policymaker) may change the type of one or more cells (e.g., sealed into green or vice versa), thus forming a scenario. The comparison against the baseline is again visualized within the 3D city visualization (see the "Editing and Comparing a Scenario against a Baseline" section).

\section{BACKGROUND}

The effects of urban heat islands as well as street canyons have been known at least since the beginning of industrialization (Howard 1818); however, it took more than a century for more formal microclimate models to be introduced (e.g., the pioneering work of (Oke 1982)). With regard to policy-making, we see two predominant types of models being used nowadays:

- Temperature models that simulate the differences in temperature of a city center versus rural areas, e.g., showing the formation of heat islands in the night time (see Damyanovic et al. (2016) for a concrete example).

- Vulnerability Indices: Coupled population/climate models showing the potential impact of the effects of heat islands on "vulnerable" parts of the population (e.g., the young or the elderly) (see Bhattacharjee (2019) for an example).

Both types of models are typically visualized as heat maps (see Figure 1). However, the data semantics might differ, i.e., the absolute temperature $\left[{ }^{\circ} \mathrm{C}\right]$, absolute or relative deviation from a mean temperature $\left[ \pm^{\circ} \mathrm{C}\right]$, or as a ratio $[0 \ldots 1]$, relative to a predefined range $\left[\min { }^{\circ} \mathrm{C} \ldots \max { }^{\circ} \mathrm{C}\right]$, are common. Our simplified simulation (see "Cellular Automaton" section) must be able to capture and reproduce these data semantics in order to be used as a surrogate model (Queipo et al. 2005).

\section{3D CITY VISUALIZATION}

OSMBuildings (Marsch 2018) is an extensible, web-based 3D city visualization (see Figure 2), which loads its data from OpenStreetMap (OSM Foundation 2004); it superimposes a 3D building geometry on top of a 2D map that is tile-based and adheres to the slippy map semantics (OSM Foundation 2004). We use the software as a basis for this work and extend it by Cellular Automaton and a policymaking user interface as described in the following sections.

\section{CELLULAR AUTOMATON}

A 2D Cellular Automaton is embedded into the $3 \mathrm{D}$ city visualization by treating the map tiles at a certain zoom level as cells. Each cell has the following properties:

- computed [ true, false ] - defaults to false

- sealedPercentage [\%], waterPercentage [\%], greenPercentage [\%] - all three default to 0

- computedType [ none, sealed, water, green ] - defaults to none

- overrideType [ null, sealed, water, green ] - defaults to null

- value [number] - defaults to 0

After allocating a cell, its cell type must be computed. For this we use the Overpass API (2019), a query interface for OpenStreetMap, which can fetch all the features within a cell's boundaries. We fetch all the features tagged as a building, road or water body, and clip these again to the cell boundaries, since Overpass does not do that automatically. Another problem is that roads are typically shown as a line and not as an area, and the "greenery" encompasses so many types of features that it is easier to assume it is everything that is not built, not a road, and not a water body. Accordingly, we compute

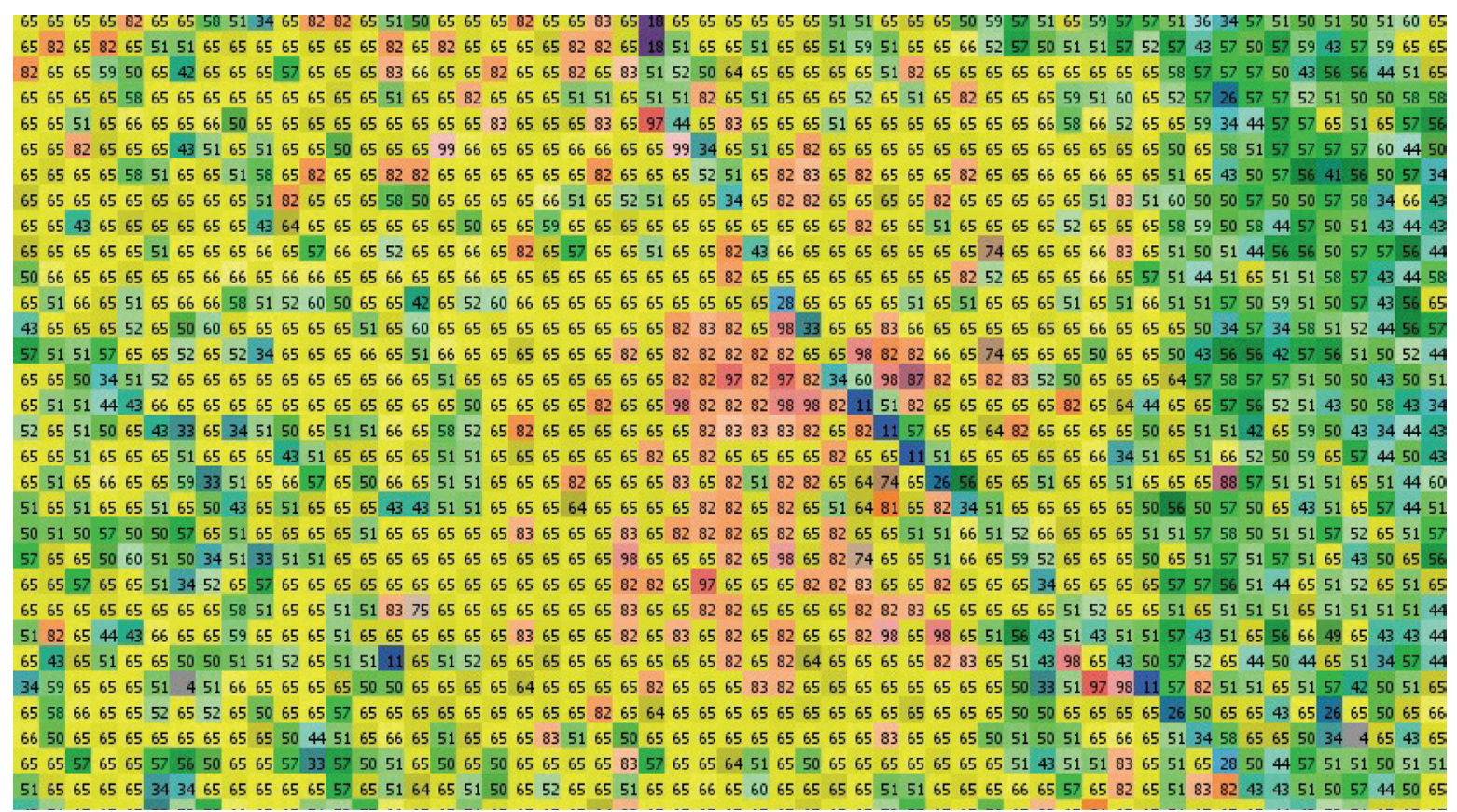

Fig. 1 Thermal image of Vienna during the night time. (Source: Figure 1 in (Damyanovic 2016):City of Vienna, MA 22; discretized to $750 \mathrm{~m} \times 750 \mathrm{~m}$ cell size; scale colors interpreted as values and translated to [0... 100]) 
- sealedPercentage $=$ (area covered by buildings + area covered by roads) / total area of the cell

- waterPercentage $=$ area covered by water $/$ total area of the cell

- greenPercentage $=1$ - (sealedPercentage + waterPercentage $)$

and then find the dominant percentage in order to get to a computed Type=sealed, water or green for that cell, which has now been computed=true. All the computed cells then enter the simulation, whose job it is to approximate simulated data based on the cell type and cell neighborhood. More specifically, we search for a cell's value, which might be (an absolute or a relative) temperature or, as in the case of our study (see the next section), a percentage of the maximal temperature.

The value is defined as

$$
\text { value }(\text { cell })=\left\{\begin{array}{rl}
v_{\text {seded }}, & \text { cell.cellType }=\text { sealed } \\
1_{\text {Grean }}, & \text { cell.cellType }=\text { green } \\
v_{\text {water }}, & \text { cell.cellType }=\text { water } \\
0, & \text { else }
\end{array}+\right.
$$$$
\sum_{i=-}^{r} \sum_{j=-}^{n_{i}(c e t h)} \frac{\text { neighborValue }\left(n_{i, j}(\text { cell })\right)}{i}
$$

where $n_{i}($ cell $)$ denotes the set of cells at distance $i$ around $c e l l, n_{i, j}($ cell $)$ is the $j$-th cell from that set, and

$$
\text { neighborValue(neighborCell })=
$$

$$
\left\{\begin{aligned}
v_{\text {sealed Neighbor }}, & \text { neighborCell.cellType }=\text { sealed } \\
v_{\text {green Neighbor }}, & \text { neighborCell.cellType }=\text { green } \\
v_{\text {water Noighbor }}, & \text { neighborCell.cellType }=\text { water } \\
0, & \text { else }
\end{aligned}\right.
$$

The value variables $\mathrm{v}_{\text {sealed }}, \mathrm{v}_{\text {green }}, \mathrm{v}_{\text {water }}, \mathrm{v}_{\text {sealedNeighbor }}, \mathrm{v}_{\text {greenNeighbor }}$, $\mathrm{v}_{\text {waterNeighbor }}$ and the inclusion radius $r$ must be fitted into a pre-step in order to resemble the outputs from a full simulation, as outlined in the next section.

\section{APPROXIMATING SIMULATED DATA - VIENNA CASE STUDY}

Starting with outputs from a full-fledged simulation or measurement, one needs to find $\mathrm{v}_{\text {sealed }}, \mathrm{v}_{\text {green }}, \mathrm{V}_{\text {water }}, \mathrm{V}_{\text {sealedNeighbor? }}$

Tab. 1 Variable values obtained through fitting.

\begin{tabular}{|c|c|c|c|c|c|c|}
\hline $\mathrm{v}_{\text {sealed }}$ & $\mathrm{v}_{\text {green }}$ & $\mathrm{v}_{\text {water }}$ & $\mathrm{v}_{\text {sealedNeighbor }}$ & $\mathrm{v}_{\text {greenNeighbor }}$ & $\mathrm{v}_{\text {waterNeighbor }}$ & $\mathrm{r}$ \\
\hline $36 \%$ & $18 \%$ & $-33 \%$ & $14 \%$ & $11 \%$ & $7 \%$ & 1 \\
\hline
\end{tabular}

$\mathrm{V}_{\text {greenNeighbor }}, \mathrm{v}_{\text {waterNeighbor }}$ and $r$, such that the deviation between each cell's approximated value (found through Equations 1 and 2) and the value computed by the full-fledged simulation (ground truth) is minimal. How this fitting process is conducted, e.g., via complete enumeration (parameter sweep) or using Genetic Algorithms, is up to the user.

Our case study was based on the temperature map of Vienna shown in Figure 1, which shows percentages between the minimum and maximum temperatures [0\%- $100 \%]$. The cells were categorized into water for temperature $<30 \%$, greenery for temperature $<$ $70 \%$, and sealed in all other cases. Through parameter sweeping, we obtained the variable values in Table 1 . The deviation between the temperature map and approximated values computed by the Cellular Automaton is given in Table 2 and visualized in Figure 3.

\section{EDITING AND COMPARING A SCENARIO AGAINST A BASELINE}

Users can edit each cell, giving it a new type (see the cell setting in the top left part of Figure 2). The updated approximation is then given as a value and visualized as the color of the cell's marker. Changes in temperature levels resulting from user interventions are furthermore shown as a gauge (see the top right part of Figure 2), which depicts the relative change: no change is visualized as a gauge pointer exactly in the middle (see again, top right in Figure 2); the lower temperature is to the left, and the higher temperature is to its right.

\section{DISCUSSION}

The fit does not account for the reductive effects of the cell neighbors. This is likely due to bad input data (a parameter sweep was done for both the negative and positive numbers; however, the data is too spatially aggregated to show the reductive effects of the greenery and water bodies on the sealed cells). As a consequence, changing a cell's type has almost no effect if it is not water (because $\mathrm{v}_{\text {water }}$ is negative).

A manually set Cellular Automata calibration that also has negative values for the neighbors might seem to be a good solution; however, one must also make sure that a Cellular Automaton with only one type of cell produces the approximate temperature in every cell (currently not the case). It might well be that one must treat the interaction between the cells depending on their type; for example, water may not be influenced by other cell neighbors, while sealed cells will react to water in its surroundings. A quick test with the parameters $\mathrm{v}_{\text {sealed }}=\mathrm{v}_{\text {sealedNeighbor }}=11.11 \%, \mathrm{v}_{\text {green }}=\mathrm{v}_{\text {greenNeighbor }}=5.55 \%, \mathrm{v}_{\text {water }}=\mathrm{v}_{\text {water- }}$ $=-11.11 \%$ and $\mathrm{r}=1$ plus the additional rule that water does not take its neighborhood into account yields much more comprehensible results both in terms of the cell temperatures as well as the impact of user interventions (see the colors of the cell markers and gauge in Figure 4).

Tab. 2 Fitting results.

\begin{tabular}{|l|l|l|l|l|l|l|l|l|l|l|}
\hline & & \multicolumn{3}{|c|}{ ground truth } & \multicolumn{3}{c|}{ approximation } & \multicolumn{3}{c|}{ deviation from ground truth } \\
\hline cell type & amount & average & $\min$ & $\max$ & average & $\min$ & $\max$ & average & $\min$ & max \\
\hline all cells & 2200 & $61.64 \%$ & $4 \%$ & $99 \%$ & $64.39 \%$ & $3 \%$ & $92 \%$ & $\pm 5.86 \%$ & $-19 \%$ & $+34 \%$ \\
\hline sealed & 218 & $84.22 \%$ & $74 \%$ & $99 \%$ & $83.52 \%$ & $72 \%$ & $92 \%$ & $\pm 4.72 \%$ & $-19 \%$ & $+12 \%$ \\
\hline green & 1963 & $59.59 \%$ & $33 \%$ & $66 \%$ & $62.78 \%$ & $54 \%$ & $74 \%$ & $\pm 5.97 \%$ & $-11 \%$ & $+34 \%$ \\
\hline water & 19 & $15.58 \%$ & $4 \%$ & $28 \%$ & $11.58 \%$ & $3 \%$ & $17 \%$ & $\pm 7.68 \%$ & $-15 \%$ & $+9 \%$ \\
\hline
\end{tabular}




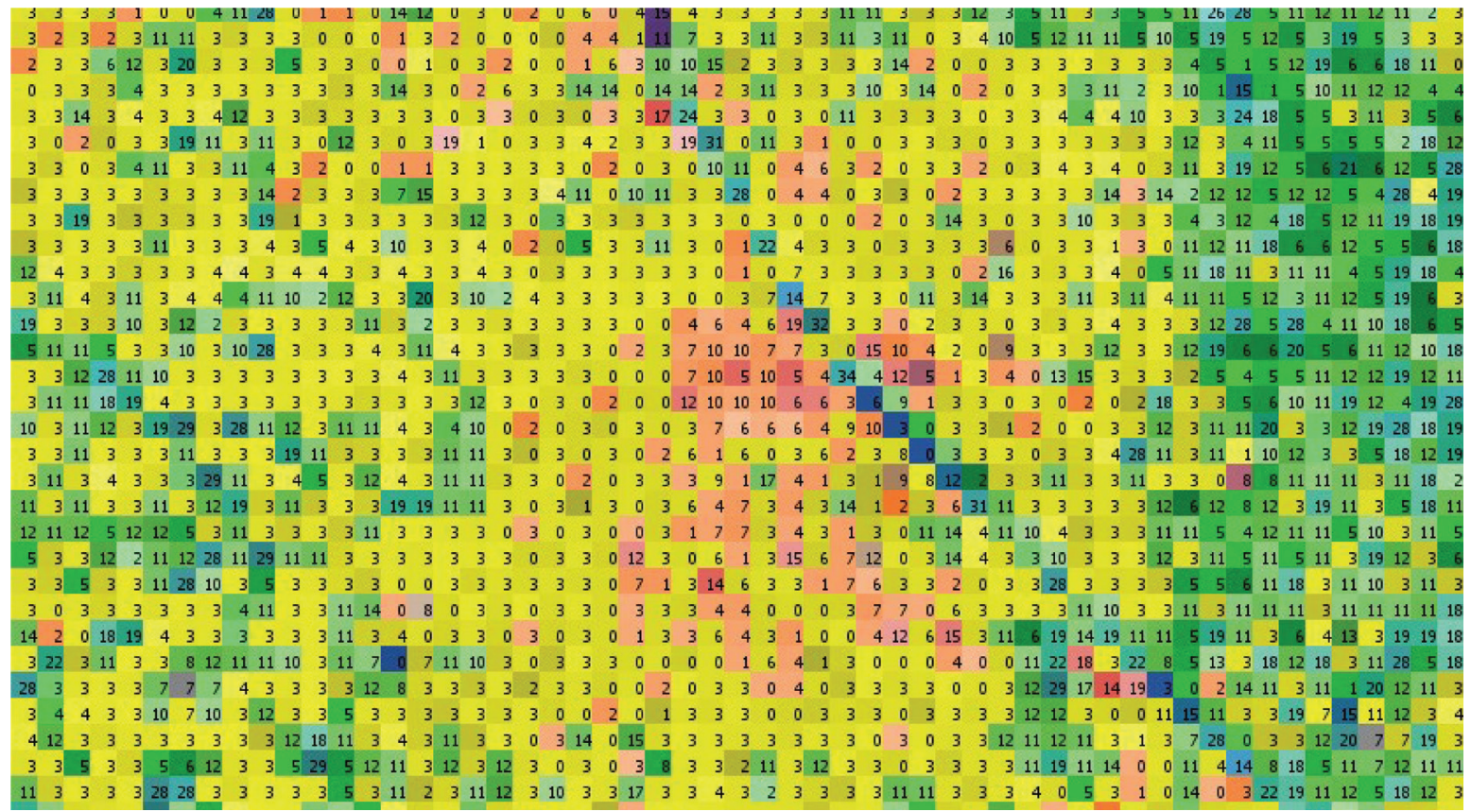

Fig. 3 Absolute deviation between the ground truth and the approximated values, in [\%].

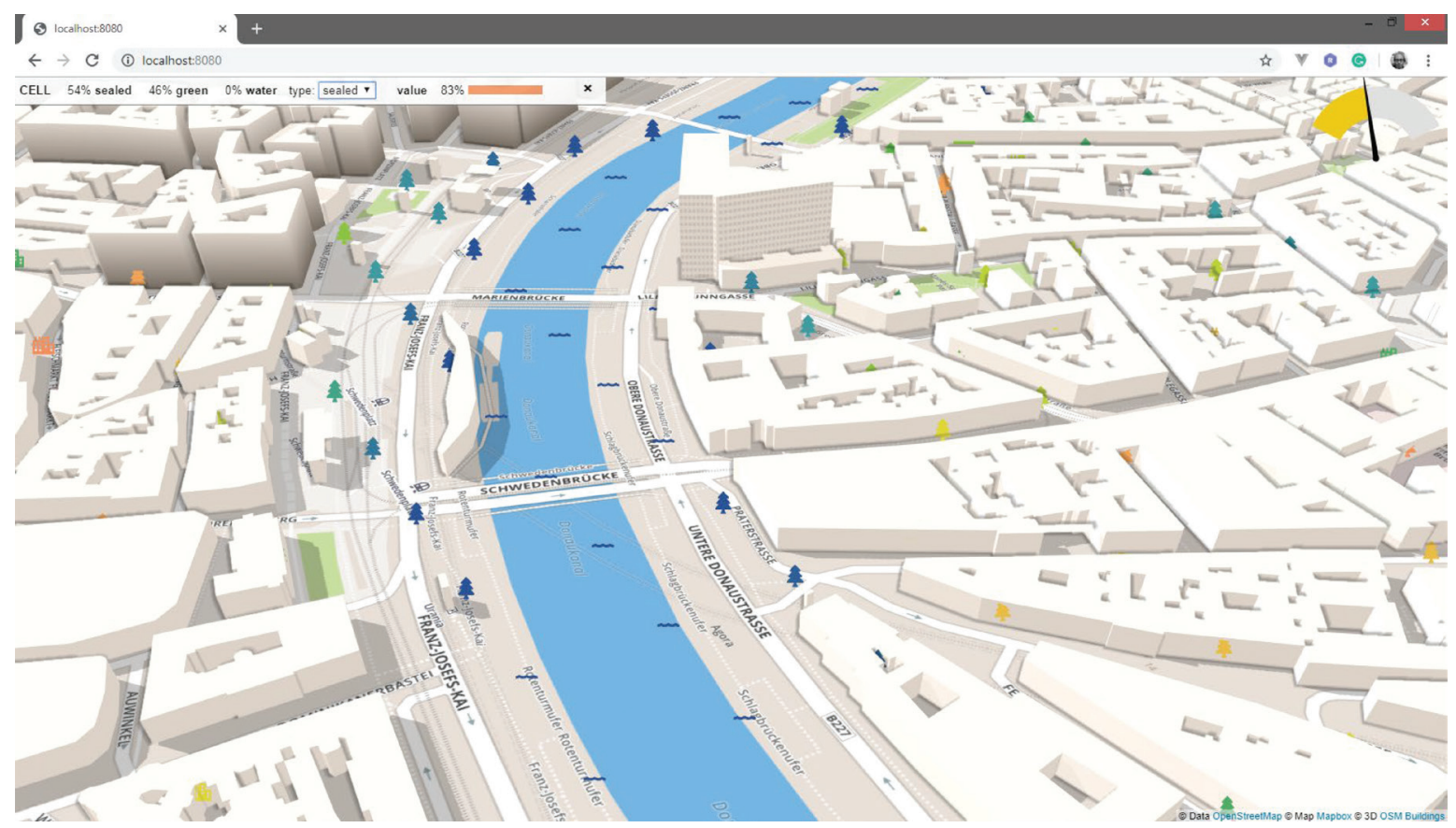

Fig. 4 Results from manually set parameters.

\section{CONCLUSIONS}

We have presented a web-based 3D simulation platform for estimating UHI effects aimed at policymakers that use a Cellular Automaton (cell types sealed, green, water) to approximate values from actual temperature measurements or a full-fledged simulation. We have also included the means for policymakers to change cell types in order to simulate urban interventions, thereby also changing the microclimate. All in all, we find that this is a promising platform for showing microclimate effects to policymakers; however, more efforts on calibration and fitting are required in the near future for a better fit to the requirements of today's decision-making. 


\section{REFERENCES}

Bhattacharjee, S. (2019) The urban heat vulnerability map of Vienna, Austria. Available online: https://www.wien.gv.at/stadtentwicklung/energie/pdf/hitzekarte-methode.pdf [accessed 8th Sept. 2019].

Bruse, M. (1999) The influences of local environmental design on microclimate. Ph.D Thesis, University of Bochum.

Bruse, M., - Fleer, H. (1998) Simulating Surface-Plant-Air Interactions Inside Urban Environments with a Three Dimensional Numerical Model, Environmental Software and Modelling 13, pg. 373-384

Howard, L. (1818) The climate of London, deduced from Meteorological observations, made at different places in the neighbourhood of the metropolis. W. Phillips, London.

Damyanovic, D., - Reinwald, F., - Brandenburg, C., - Allex, B., - Gantner, B., - Morawetz, U., - Preiss, J. (2016) Pilot action city of Vienna - UHI-STRAT Vienna, in Francesco Musco (ed.), Counteracting Urban Heat Island Effects in a Global Climate Change Scenario, Springer, pg. 257-280.

Marsch, J. (2018) OSMBuildings (software). Available online: https://osmbuildings.org [accessed 1st May 2019].
Oke, T. R. (1982) The energetic basis of the urban heat island. Quarterly Journal of the Royal Meteorological Society 108 (455), pp. $1-24$.

OSM Foundation (2004) OpenStreetMap (data repository). Available online: https://www.openstreetmap.org [accessed 1st May 2019].

OSM Foundation (2004) Slippy Map Tilenames (web page). Available online: https://wiki.openstreetmap.org/wiki/Slippy_ map_tilenames [accessed 1st May 2019].

Overpass API (2019) (web page) Available online: http://overpass-api.de/ [accessed August 2019].

Queipo, N. V., - Haftka, R. T., - Shyy, W., - Goel, T., - Vaidyanathan, R., - Tucher, P. K. (2005) Surrogate-based Analysis and Optimization, Progress in Aerospace Sciences 41, pg. 1-28.

U.S. Environment Protection Agency (2019) Heat Island Effect. Available online: https://www.epa.gov/heat-islands [accessed 1st May 2019]. 